\title{
Enhancement of ENSO's persistence barrier by biennial variability in a coupled atmosphere-ocean general circulation model
}

\author{
Jin-Yi Yu \\ Department of Earth System Science, University of California, Irvine, Irvine, California, USA
}

Received 9 May 2005; revised 2 June 2005; accepted 8 June 2005; published 15 July 2005.

[1] Possible causes of the spring persistence barrier in ENSO sea surface temperature anomalies are examined using a coupled atmosphere-ocean general circulation model (CGCM). Our study indicates that the persistence barrier is significantly enhanced when both Pacific and Indian Ocean couplings are included in the CGCM, compared to the simulation that includes only the Pacific Ocean coupling. ENSO's variance, phase locking to the annual cycle, and biennial variability are also increased in the Indo-Pacific Run. Further analysis reveals that the overall amplitude of ENSO is not a primary factor in determining the strength of the persistence barrier, rather, it is the amplitude of the biennial component of ENSO affecting the barrier the most. The persistence barrier is consistently strong (weak) when biennial ENSO variability is large (small). No such a clear relationship is found between the strength of the barrier and the amplitude of the low-frequency ( $3-5$ years) component of ENSO. This modeling study demonstrates that the biennial component of ENSO is one major mechanism responsible for the spring persistence barrier and that interactions between the tropical Pacific Ocean and Indian Ocean-monsoon could enhance the biennial component of ENSO. Citation: Yu, J.-Y. (2005), Enhancement of ENSO's persistence barrier by biennial variability in a coupled atmosphere-ocean general circulation model, Geophys. Res. Lett., 32, L13707, doi:10.1029/2005GL023406.

\section{Introduction}

[2] The presence of a spring barrier in El Niño Southern Oscillation (ENSO) is well known. Autocorrelation analyses with various ENSO indices, such as the NINO3 sea surface temperature (SST) anomalies, Southern Oscillation pressure differences, and central Pacific rainfall anomalies, show sharp declines in the correlation coefficients in boreal spring [Troup, 1965; Wright, 1979; Webster and Yang, 1992; Torrence and Webster, 1998; Clarke and Van Gorder, 1999]. The cause of this persistence barrier has not yet been fully understood, and various hypotheses have been suggested. Wright [1979] and Nicholls [1979] suggested that it may be related to the sign change of atmosphere-ocean feedbacks from one season to another. Webster and Yang [1992] stated that the weak Walker circulation in spring produces the barrier by allowing random perturbations to disrupt the persistence of ENSO anomalies. Lau and Yang [1996] demonstrated evidence that indicates a close relationship between the barrier and the intensity of the monsoon. Their result suggested that the Asian monsoon is another important factor to influence the persistence barrier.

Copyright 2005 by the American Geophysical Union. 0094-8276/05/2005GL023406\$05.00
Torrence and Webster [1998] proposed the phase locking of ENSO to the annual cycle as the cause of the barrier. Their argument is based on the fact that ENSO tends to transit from one state to another in spring, and ENSO's variance and signal-to-noise ratio are the lowest during this season, thus reducing the ENSO persistence in spring. ENSO is known to have a low-frequency ( $3-5$ years) and a biennial ( 2 years) component [Rasmusson and Carpenter, 1982; Rasmusson et al., 1990; Wang and Wang, 1996; Gu and Philander, 1997]. Clarke and Van Gorder [1999] emphasized the importance of the biennial component for producing the barrier. They argued that the biennial component always has its phase transitions occurring in the spring, and hence is responsible for the phase locking and the spring persistence barrier of ENSO.

[3] In this study, we conducted two experiments with the University of California Los Angeles (UCLA) CGCM (coupled atmosphere-ocean general circulation model) to contrast the relationship among the persistence barrier, phase locking, and biennial variability of ENSO. These two experiments differ in their interactive ocean domains in the CGCM: one includes only the tropical Pacific Ocean in the OGCM, the other includes both the tropical Pacific and Indian Oceans in the model domain. As shown later, the addition of an interactive Indian Ocean affects the CGCM simulations of ENSO, and therefore offers an opportunity to gain insight into the cause of the persistence barrier.

\section{Model and Experiments}

[4] The CGCM used in this study consists of the UCLA AGCM [Mechoso et al., 2000] and the Geophysical Fluid Dynamics Laboratory (GFDL) Modular Ocean Model (MOM) [Bryan, 1969; Cox, 1984] (see Yu and Mechoso [2001] for a description of the CGCM). The AGCM is global with a horizontal resolution of $4^{\circ}$ latitude by $5^{\circ}$ longitude and 15 levels in the vertical. The oceanic model has a longitudinal resolution of $1^{\circ}$, a latitudinal resolution varying gradually from $1 / 3^{\circ}$ between $10^{\circ} \mathrm{S}$ and $10^{\circ} \mathrm{N}$ to almost $3^{\circ}$ at $50^{\circ} \mathrm{N}$ and $50^{\circ} \mathrm{S}$, and 27 layers in the vertical. In the first experiment (Pacific Run), the interactive ocean domain of the CGCM covers the Pacific Ocean from $30^{\circ} \mathrm{S}$ to $50^{\circ} \mathrm{N}$ and from $130^{\circ} \mathrm{E}$ to $70^{\circ} \mathrm{W}$. In the second experiment (Indo-Pacific Run), the domain is extended westward to cover both the Indian and Pacific Oceans from $30^{\circ} \mathrm{E}$ to $70^{\circ} \mathrm{W}$. The sea/land masks of these two experiments can be found in $Y u$ et al. [2002, Figure 1]. Outside the interactive oceanic domains, SSTs and sea ice distributions for the AGCM are prescribed based on monthly climatologies. Inside the ocean model domains, SSTs poleward of $20^{\circ} \mathrm{S}$ and $30^{\circ} \mathrm{N}$ are relaxed toward their climatological values. Eighty years of simula- 

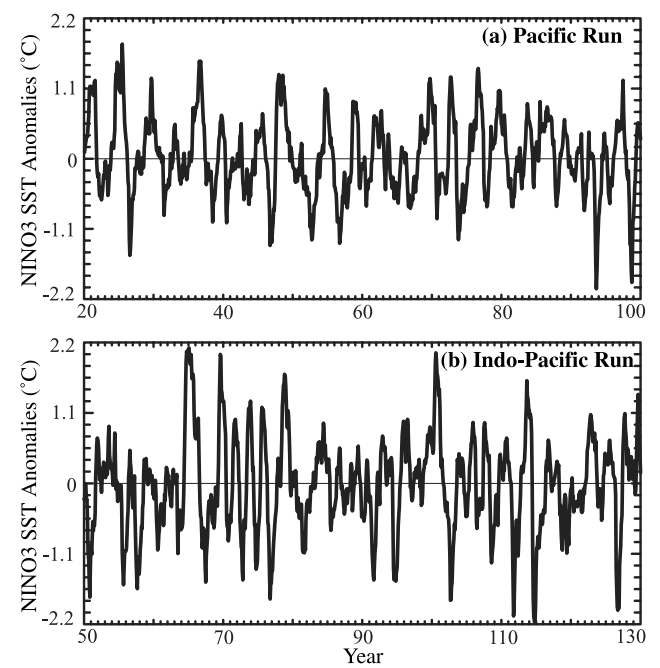

Figure 1. NINO3 SST anomalies calculated from (a) Pacific Run and (b) Indo-Pacific Run. Values shown are monthly departures from their corresponding seasonal cycles.

tions are analyzed in this study. Both runs produce a reasonable SST climatology in the Pacific and Indian Oceans, similar to those shown in Yu et al. [2002, Figure 1].

\section{Simulated ENSO Variability, Persistence, and Phase Locking}

[5] Figure 1 shows the variations in NINO3 SST anomalies produced by the two experiments. The anomalies are obtained by removing the respective seasonal cycles from the NINO3 time series. The Indo-Pacific Run produces larger ENSO SST anomalies than the Pacific Run. The standard deviation of NINO3 is $0.74^{\circ} \mathrm{C}$ for the Indo-Pacific Run and $0.59^{\circ} \mathrm{C}$ for the Pacific Run. In addition, the ENSO in the Indo-Pacific Run seems to be more irregular and shows stronger decadal variations than the Pacific Run. Thus, the addition of an interactive Indian Ocean in the CGCM tends to increase the amplitude and decadal variations of ENSO. These impacts of the Indian Ocean on ENSO simulations are consistent with those reported by Yu et al. [2002].

[6] Figures 2a and 2b show the simulated ENSO persistence from these two experiments. The lagged autocorrelation coefficients of NINO3 are calculated with the base month placed in January, February, March, ..., and December, respectively, for each of the twelve curves shown in

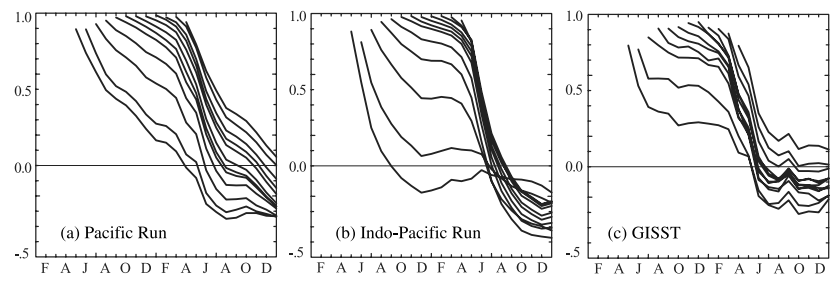

Figure 2. Lagged autocorrelation coefficients of NINO3 SST anomalies calculated from (a) Pacific Run, (b) IndoPacific Run, and (c) GISST data. The curves are shift to line up their one-month lag with the calendar month on the abscissa. each panel. The correlation curves are shifted to line up their one-month lag with the calendar month shown on the abscissa. A persistence barrier is manifested as sharp declines of the correlation curves in particular calender months. For the Pacific Run, Figure 2a shows a more gradual decrease in the correlations and a weaker dependence of the decline on calendar months. This experiment produces a weaker spring persistence barrier than the observed (Figure 2c). Correlation coefficients in Figure 2c are calculated using observed SSTs from 1901 to 2000 based on the Global Sea-Ice and Sea Surface Temperature Data Set (GISST) [Rayner et al., 1996]. In the Indo-Pacific Run (Figure 2b), the spring barrier is stronger and more realistic, with a rapid decline in the correlations occurring in MarchMay for most of the twelve curves. Figure 2 indicates that the addition of the Indian Ocean coupling can improve the CGCM simulation of the spring persistence barrier.

[7] According to the hypothesis put forward by Torrence and Webster [1998] and Clarke and Van Gorder [1999], the persistence barrier is related to ENSO's phase locking to the annual cycle. The stronger the phase locking is, the more obvious the persistence barrier becomes. To examine the phase locking, we show in Figure 3 the standard deviation of NINO3 SST anomalies as a function of the calendar month calculated from the two experiments. In the Pacific Run, the ENSO variance is minimum in spring similar to the observed timing, and maximum in summer earlier than the observed timing (maximum in winter). In the Indo-Pacific Run, ENSO variance is increased during fall and winter. As a result, its ENSO variance reaches a minimum in spring and a maximum in late fall and early winter, closer to the observed timing. In addition, the difference between the maximum and minimum variance is larger in the IndoPacific Run than in the Pacific Run. These comparisons indicate that an enhanced persistence barrier is accompanied with a strong phase locking. Furthermore, both the timing and the amplitude of the standard deviations are important to determine the phase locking.

\section{Relative Importance of ENSO Low-Frequency and Biennial Components to the Persistence Barrier}

[8] We perform power spectral analysis to examine the biennial and low-frequency components in the simulated

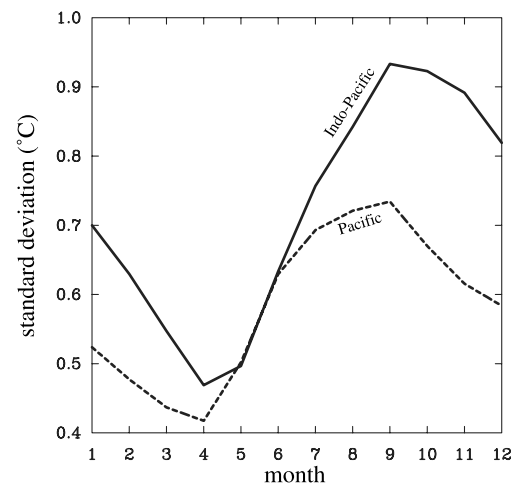

Figure 3. Standard deviations of NINO3 SST anomalies as a function of calendar month calculated from Pacific Run (dashed) and Indo-Pacific Run (solid). 

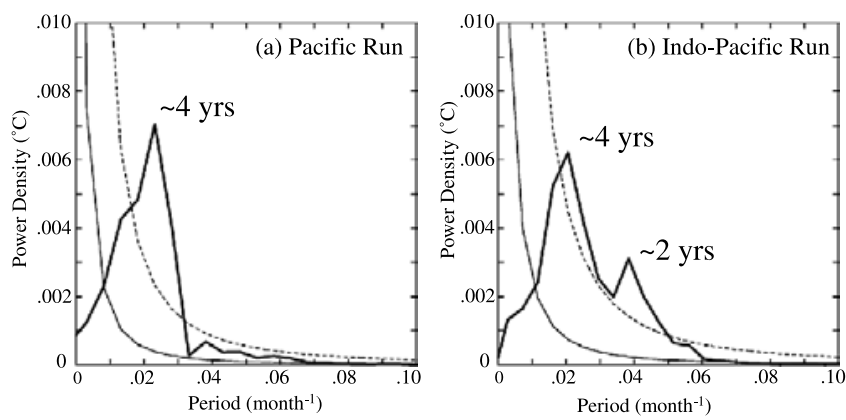

Figure 4. Power spectra of NINO3 SST anomalies calculated from (a) Pacific Run and (b) Indo-Pacific Run. Dashed lines indicate the 95\% significance level.

ENSO. Figure 4 shows the power spectra of the simulated NINO3 SST anomalies. In the Pacific Run (Figure 4a), the simulated ENSO is dominated by the low-frequency component with a distinct spectral peak near 4 years. The power near the 2-year period is very small. In the Indo-Pacific Run (Figure 4b), the simulated ENSO is still dominated by the near 4-year period, however, the amplitude of the biennial component has significantly increased and becomes the second largest peak in the spectrum. This increase suggests that there indeed exits a positive relationship between ENSO's biennial variability and its persistence barrier. The enhancement of the biennial component in the IndoPacific Run may be associated with the inter-basin interactions between the Pacific and Indian Oceans. The causes is being investigated and beyond the scope of this paper.

[9] We apply the wavelet analysis of Torrence and Compo [1998] to the NINO3 SST anomalies of the IndoPacific Run. The wavelet power is then averaged between 15-35 months to represent the amplitude of the biennial component and between 40-60 months for the lowfrequency component. Figure 5 shows the amplitude variations of these two components. Based on the amplitudes, we select three decades (Decade 2, 4, and 5) with strong biennial ENSO and three decades (Decade 1, 3, and 4) with

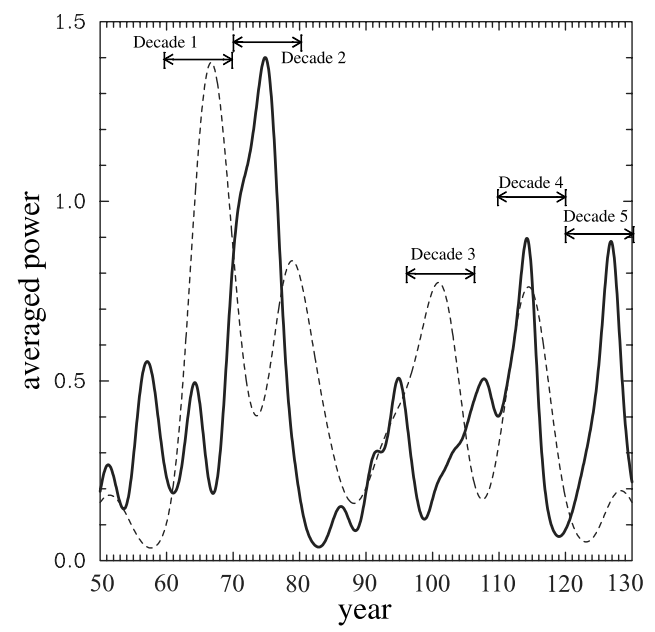

Figure 5. Variations of average wavelet power for the lowfrequency (dashed) and biennial (solid) ENSO components of the Indo-Pacific Run. The decades indicated correspond to those of Table 1.
Table 1. Decades With Different Strength of Biennial and Low-Frequency ENSO

\begin{tabular}{cccccc}
\hline $\begin{array}{c}\text { Periods } \\
\text { (years })\end{array}$ & $\begin{array}{c}\text { Decade 1 } \\
(60-70)\end{array}$ & $\begin{array}{c}\text { Decade 2 } \\
(71-80)\end{array}$ & $\begin{array}{c}\text { Decade 3 } \\
(96-106)\end{array}$ & $\begin{array}{c}\text { Decade 4 } \\
(110-120)\end{array}$ & $\begin{array}{c}\text { Decade 5 } \\
(121-130)\end{array}$ \\
\hline $\begin{array}{c}\text { Biennial } \\
\text { Low- } \\
\text { frequency }\end{array}$ & weak & $\begin{array}{c}\text { very strong } \\
\text { weak }\end{array}$ & $\begin{array}{c}\text { weak } \\
\text { strong }\end{array}$ & $\begin{array}{c}\text { strong } \\
\text { strong }\end{array}$ & $\begin{array}{c}\text { strong } \\
\text { weak }\end{array}$ \\
\hline
\end{tabular}

strong low-frequency ENSO for analysis. Decade 4 is overlapped in both selections. Table 1 lists the five decades selected and the strength of their ENSO components.

[10] Figure 6 shows the persistence of NINO3 SST anomalies in these five decades. All the three decades with a large biennial component produce strong persistence barriers in spring. Decade 2 shows a particularly strong barrier. All twelve curves of correlations drop sharply in April-May. Their correlation coefficients have values close to 1.0 before the drop and close to -0.8 afterwards. This pattern of persistence is close to the one predicted by Clarke and Van Gorder [1999] for a purely biennial oscillation with phase transitions in May. They showed that the correlation coefficients switch abruptly from 1.0 to -1.0 in May. Our Decade 2 is a close approximation to their pure biennial oscillation case.

[11] The comparison of Decade 4 and 5 is interesting because they both have a similarly large biennial component but very different amplitudes in the low-frequency component. The low-frequency component is small in Decade 5 but as large as the biennial component in Decade 4 . The similarity in the persistence barriers shown in these two decades (Figures $6 \mathrm{~b}$ and $6 \mathrm{c}$ ) suggests that the importance of the biennial component to the persistence barrier is not affected much by the presence of a large lowfrequency component. The weakness of persistence barriers shown in Decade 1 and 3, which are another two decades with a large low-frequency component, further demonstrates that the low-frequency component is less important to the persistence barrier than the biennial component. It is also interesting to compare Decade 1 and 2. They are the two decades that have the largest

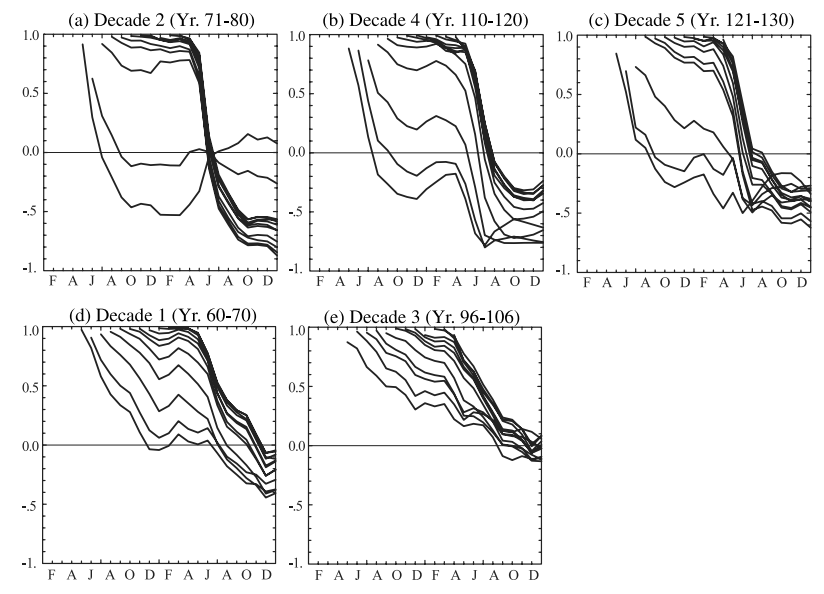

Figure 6. Sam as Figure 2, but for (a) Decade 2 (years 71-80), (b) Decade 4 (110-120), (c) Decade 5 (121-130), (d) Decade 1 (60-70), and (e) Decade 3 (96-106) of the Indo-Pacific Run. 
ENSO variability in the Indo-Pacific Run, but one is dominated by the low-frequency component and the other by the biennial component. The strong barrier in Decade 2 and the weak barrier in Decade 1 imply that the overall amplitude of ENSO is not a primary factor to determine the persistence barrier. It is the amplitude of the biennial component of ENSO that matters.

\section{Summary and Discussion}

[12] We have used two CGCM experiments to examine the possible causes of the persistence barrier in ENSO's SST anomalies. This is accomplished by comparing CGCM simulations in which SSTs in the Indian Ocean are either prescribed or determined by coupling with the atmosphere. Our results show that biennial ENSO variability is significantly enhanced when both Pacific and Indian Ocean couplings are included in the CGCM, compared to the simulation that includes only the Pacific Ocean coupling. Accompanying this, the spring persistence barrier and phase locking of ENSO are enhanced and become more realistic. Wavelet analysis is used to examine the amplitude variations of the biennial and low-frequency ENSO components and their relationships with these enhancements. It is found that the spring persistence barrier is strong in the decades with strong biennial ENSO and is weak in the decades with weak biennial ENSO. No such a clear relationship is found between the strength of the barrier and the amplitude of the low-frequency ENSO component. We emphasize that a large ENSO variance does not guarantee a strong persistence barrier. It is the amplitude of biennial component that affects the strength of the persistence barrier. Our analysis also showed that the presence of the biennial component produces a more realistic phase locking of ENSO in the CGCM.

[13] Our modeling results support the hypotheses that the spring persistence barrier is a result of the phase locking of ENSO [Torrence and Webster, 1998; Clarke and Van Gorder, 1999] and that the biennial ENSO component is crucial to the phase locking [Clarke and Van Gorder, 1999]. This study also suggests that the Indian Ocean coupling plays a key role in producing the biennial component of ENSO. The mechanisms for this are not yet understood, but are being studied. The tropospheric biennial oscillation (TBO) in the Indian Ocean-monsoon may be involved in the enhancement of biennial ESNO variability. Our recent study [Yu et al., 2003] suggested that the TBO is related to atmosphere-ocean coupling in both Indian and Pacific Oceans. One possibility is that variations in the strength of the monsoon system affect Pacific trade winds, and consequently, the period and magnitude of ENSO [Barnett, 1984; Wainer and Webster, 1996; Clarke et al., 1998; Chung and Nigam, 1999; Lau and Yang, 1996; Lau and Wu, 2001; Kim and Lau, 2001]. This modeling study adds evidence to the notion that the biennial climate variability in the tropics involves inter-basin interactions between tropical Pacific and Indian Oceans. It should be noted that the conclusions reported in this study is based on one model and can be model dependent.

[14] Acknowledgments. This work was supported by a grant from NOAA OGP's CLIVAR- Pacific Program (NA16GP1016) and a grant from NASA's SENH Program (NAG5-13248) through a contract from UCLA.
The author thanks Dr. Hua Hu and two anomalous reviewers for their comments that improve this paper. Model integrations and analyses were performed at the San Diego Supercomputer Center (SDSC) and UC Irvine's Earth System Modeling Facility which is funded by the National Science Foundation. The wavelet analysis code is downloaded from University of Colorado at http://paos.colorado.edu/research/wavelets/.

\section{References}

Barnett, T. P. (1984), Interaction of the monsoon and Pacific trade wind system at interannual time scales, Mon. Weather Rev., 112, 23802387.

Bryan, K. (1969), A numerical method for the study of the circulation of the world ocean, J. Comput. Phys., 4, 347-376.

Chung, C., and S. Nigam (1999), Asian summer monsoon-ENSO feedback on the Cane-Zebiak model ENSO, J. Clim., 12, 2787-2807.

Clarke, A. J., and S. Van Gorder (1999), The connection between the boreal spring Southern Oscillation persistence barrier and biennial variability, J. Clim., 12, 610-620.

Clarke, A. J., X. Liu, and S. V. Gorder (1998), Dynamics of the biennial oscillation in the equatorial Indian and far western Pacific Oceans, J. Clim., 11, 987-1001.

Cox, M. D. (1984), A primitive equation three-dimensional model of the ocean, Tech. Rep. 1, Geophys. Fluid Dyn. Lab. Ocean Group, Princeton, N. J.

Gu, D., and S. G. H. Philander (1997), Interdecadal climate fluctuations that depend on exchanges between tropics and extratropics, Sciences, 275, $805-807$.

Kim, K.-M., and K.-M. Lau (2001), Dynamics of monsoon-induced biennial variability in ENSO, Geophys. Res. Lett., 28, 315-318.

Lau, K.-M., and S. Yang (1996), The Asian monsoon and predictability of the tropical ocean-atmosphere system, Q. J. R. Meteorol. Soc., 122, 945957.

Lau, K.-M., and H.-T. Wu (2001), Principal modes of rainfall-SST variability of the Asian summer monsoon: A reassessment of the monsoonENSO relationship, J. Clim., 14, 2880-2895.

Mechoso, C. R., J.-Y. Yu, and A. Arakawa (2000), A coupled GCM pilgrimage: From climate catastrophe to ENSO simulations, in General Circulation Model Development: Past, Present, and Future, Int. Geophys. Ser, vol. 70, edited by D. A. Randall, pp. 539-575, Elsevier, New York.

Nicholls, N. (1979), A simple air-sea interaction model, Q. J. R. Meteorol. Soc., 105, 93-105.

Rasmusson, E. M., and T. H. Carpenter (1982), Variations in tropical sea surface temperature and surface wind fields associated with the Southern Oscillation/El Niño, Mon. Weather Rev., 110, 354-384.

Rasmusson, E. M., X. Wang, and C. F. Ropelewski (1990), The biennial component of ENSO variability, J. Mar. Syst., 1, 71-96.

Rayner, N. A., E. B. Horton, D. E. Parker, C. K. Folland, and R. B. Hackett (1996), Version 2.2 of the Global Sea-Ice and Sea Surface Temperature Data Set, 1903-1994, Clim. Res. Tech. Note 74, Met Office, Bracknell, U. K.

Torrence, C., and G. P. Compo (1998), A practical guide to wavelet analysis, Bull. Am. Meteorol. Soc., 79, 61-78.

Torrence, C., and P. J. Webster (1998), The annual cycle of persistence in the El Niño/Southern Oscillation, Q. J. R. Meteorol. Soc., 124, 19852004.

Troup, A. J. (1965), The "southern oscillation," Q. J. R. Meteorol. Soc., 91, 490-506.

Wainer, I., and P. J. Webster (1996), Monsoon-ENSO interaction using a simple coupled ocean-atmosphere model, J. Geophys. Res., 101, 25,59925,614 .

Wang, B., and Y. Wang (1996), Temporal structure of the Southern Oscillation as revealed by waveform and wavelet analysis, J. Clim., 9, 15861598 .

Webster, P. J., and S. Yang (1992), Monsoon and ENSO: Selectively interactive systems, Q. J. R. Meteorol. Soc., 118, 877-925.

Wright, P. B. (1979), Persistence of rainfall anomalies in the central Pacific, Nature, 277, 371-374.

Yu, J.-Y., and C. R. Mechoso (2001), A coupled atmosphere-ocean GCM study of the ENSO cycle, J. Clim., 14, 2329-2350.

Yu, J.-Y., C. R. Mechoso, J. C. McWilliams, and A. Arakawa (2002), Impacts of the Indian Ocean on the ENSO cycle, Geophys. Res. Lett., 29(8), 1204, doi:10.1029/2001GL014098.

Yu, J.-Y., S.-P. Weng, and J. D. Farrara (2003), Ocean roles in the TBO transitions of the Indian-Australian monsoon system, J. Clim., 16, 30723080 .

J.-Y. Yu, Department of Earth System Science, University of California, Irvine, Irvine, CA 92697-3100, USA. (jyyu@uci.edi) 